\title{
Formally exact quantization condition for nonrelativistic quantum systems
}

\author{
Yong-Cheng Ov and Zhuang-Qi Cao, and Qi-Shun Shen \\ Department of Physics, Shanghai Jiao Tong University, Shanghai 200240, People's Republic of China
}

\begin{abstract}
Based on the standard transfer matrix, a formally exact quantization condition for arbitrary potentials, which outflanks and unifies the historical approaches, is derived. It can be used to find the exact bound-state energy eigenvalues of the quantum system without solving an equation of motion for the system wave functions.
\end{abstract}

PACS numbers:

In general an exact solution for many quantum mechanical problem is unavoidable and one is forced to resort to some types of approximation technique. The Wentzel-Kramers-Brillouin(WKB) approximation, based on the wave function expansion in powers of $\hbar$, is studied and applied frequently in many fields. Apparently, it only remains valid for the short-waves and then plays a role of a bridge between classical and quantum mechanics. Neglecting of the higher order of $\hbar$ directly leads to failing to describe the wave function at the classical turning points, together with inaccurate energy eigenvalues in the lower bound states. Langer [1] introduced an approximation based on Bessel function, coming across the same problems as the WKB approximation. Rather than in terms of the exponential function, Miller 2] proposed a method by proper choice of the arbitrary equation. Friedrich [3] believed the phase shift at the tuning point is energy-dependent with nonintegral Maslov indices in the limit of the long-waves. Recently, the structure of supersymmetric quantum mechanics motivates a modified semiclassical quantization condition for one-dimensional Hamiltonians. But the supersymmetric WKB(SWKB) method only reproduces the exact energy eigenvalues for translationally shape-invariant potentials whose groundstate wave function is analytically known [4] [5]. When the two or three-dimensional potentials are dealt with, Langer-like corrections [1] is needed, which is exact only for solvable spherically symmetric potentials. All above methods are unable to give an exact quantization condition for arbitrary nonrelativistic quantum systems.

In this paper, from a different starting point, we first take the standard transfer matrix as the basis of analysis, and deduce the exact quantization condition in which the scattering-led phase shift recovers the neglecting of series of higher order $\hbar$ in the WKB approximation. However, the scattering-led phase shift term is of discrete sum formalism, furthermore, it is a bit purer mathematical and lacks of physical insights. But the defined equivalent attenuation vector is recognized to be nothing more than $-\psi^{\prime}(x) / \psi(x)$, so that an elegant integral formalism of the scattering-led phase shift can be instead of the discrete sum formalism. A Riccati equation is obtained, which is associated the wave function with the scattering-led phase shift, and we show the quantization condition outflanks and unifies the historical approaches, leading directly to the exact eigenvalues and the exact eigenfunction so that we can define an exact real quantum action-angle variable $J(E)$ determining the energy eigenvalues.

Without loss of generality, we assume a potential with two classical turning points $x_{L}$ and $x_{R}$ which are determined from $V\left(x_{L}\right)=V\left(x_{R}\right)=E$. Far from the two points, we truncate the potential at $x_{C}$ and $x_{D}$. In order to employ the transfer matrix, $\left[x_{C}, x_{D}\right]$ is divided into $l+f+g$ equal section layers with width $h$, letting $\left[x_{C}, x_{L}\right]$ have $l$ section layers, $\left[x_{L}, x_{R}\right]$ have $f$ section layers and $\left[x_{R}, x_{D}\right]$ have $g$ section layers. In each section layer, the potential can be regarded as constant. Hence the transfer matrix of $j$ th layer can be written as

$$
M_{j}=\left[\begin{array}{cc}
\cos \left(\kappa_{j} h\right) & -\frac{1}{\kappa_{j}} \sin \left(\kappa_{j} h\right) \\
\kappa_{j} \sin \left(\kappa_{j} h\right) & \cos \left(\kappa_{j} h\right)
\end{array}\right]
$$

where $k_{j}$ is defined as $\hbar \kappa_{j}=\sqrt{2 m\left[E-V_{j}\right]}$ and the subscript $j$ is an integer ranging from 1 to $l+f+g . V_{j}$ stands for the potential energy at the $j$ th section layer, $m$ is the particle mass and $E$ is the energy eigenvalue. Using the boundary condition that the wave function $\psi(x)$ and its first derivative $\psi^{\prime}(x)$ are continuous at the boundary between two neighboring section layers, the matrix equation is given

$$
\left[\begin{array}{c}
\psi\left(x_{C}\right) \\
\psi^{\prime}\left(x_{C}\right)
\end{array}\right]=\prod_{j=1}^{l+f+g} M_{j}\left[\begin{array}{c}
\psi\left(x_{D}\right) \\
\psi^{\prime}\left(x_{D}\right)
\end{array}\right] .
$$

The right- and left-hand side in Eq.(2) are simultaneously multiplied by the matrix $\left[-\psi^{\prime}\left(x_{C}\right), \psi\left(x_{C}\right)\right]$ and divided by $\psi\left(x_{C}\right) \psi\left(x_{D}\right)$, then Eq. (2) is changed to the form

$$
\left[\begin{array}{ll}
-\frac{\psi^{\prime}\left(x_{C}\right)}{\psi\left(x_{C}\right)} & 1
\end{array}\right] \prod_{j=1}^{l+f+g} M_{j}\left[\begin{array}{c}
1 \\
\frac{\psi^{\prime}\left(x_{D}\right)}{\psi\left(x_{D}\right)}
\end{array}\right]=0 .
$$

The following analysis is based on the section layer width $h \rightarrow 0$, the mass $m=1$ and Planck's constant $\hbar=1$. We define $P_{C}=-\psi^{\prime}\left(x_{C}\right) / \psi\left(x_{C}\right)$ and $P_{D}=\psi^{\prime}\left(x_{D}\right) / \psi\left(x_{D}\right)$. Making use of the transfer characteristic of matrix in Eq.(3), the values of 
$-\psi^{\prime}(x) / \psi(x)$ are calculated at any position $x$. After algebraic manipulations [] $\underline{6}$, Eq.(3) is equivalent to

$$
\begin{aligned}
& \sum_{w=l+1}^{l+f} \kappa_{w} h+\delta(n) \\
= & n \pi+\arctan \left(\frac{P_{l}}{\kappa_{l+1}}\right)+\arctan \left(\frac{P_{l+f+1}}{\kappa_{l+f}}\right),
\end{aligned}
$$

where $\delta(n)$ is determined from

$$
\delta(n)=\sum_{i=l+1}^{l+f-1}\left[\arctan \left(\frac{P_{i+1}}{\kappa_{i+1}}\right)-\arctan \left(\frac{P_{i+1}}{\kappa_{i}}\right)\right],
$$

with

$$
P_{j}=\kappa_{j}\left[\arctan \left(\frac{P_{j+1}}{\kappa_{j}}\right)-\kappa_{j} h\right],
$$

and $P_{j}$ are subject to the boundary condition $P_{0}=$ $P_{C}, P_{l+f+g+1}=P_{D}$. From Eq.(5), we know that if the potential is nonconstant, $\delta(n)$ is nonzero, so that $\delta(n)$ can be interpreted as the scattering-led phase shift which is in general energy-dependent or quantum number $n$ dependent. It is clear that $\kappa_{l+1}=\sqrt{2\left[E-V_{l+1}\right]} \rightarrow$ 0 and $\kappa_{l+f}=\sqrt{2\left[E-V_{l+f+1}\right]} \rightarrow 0$, while $P_{l}$ and $P_{l+f+1}$ is positive and finite for bound states, so $\arctan \left(P_{l} / \kappa_{l+1}\right)=\arctan \left(P_{l+f+1} / P_{l+f}\right)=\pi / 2$ that is the energy-independent phase shift at the turning points. If a wave is reflected at $x_{L}$ where $V\left(x_{L}\right) \rightarrow \infty$, meanwhile, $P_{l} \rightarrow+\infty$ and $\kappa_{l+1}$ is a finite positive number, so the half phase shift at $x_{L} \arctan \left(P_{l} / \kappa_{l+1}\right)$ is also exactly equal to the same $\pi / 2$ as that at the turning point. The first term in Eq.(4) can be integrated as $\sum_{w=l+1}^{l+f} \kappa_{w} h=$ $\int_{x_{L}}^{x_{R}} \kappa d x$. Given that Eq.(6) is equivalent to $\kappa_{j} h=$ $\arctan \left(P_{j+1} / \kappa_{j}\right)-\arctan \left(P_{j} / \kappa_{j}\right)$, and the derivative of $\arctan (P / \kappa)$, namely, $d[\arctan (P / \kappa)] / d x=$ $(\kappa d P-P d \kappa) /\left(\kappa^{2}+P^{2}\right)$, we have

$$
\arctan \left(\frac{P_{j+1}}{\kappa_{j}}\right)-\arctan \left(\frac{P_{j}}{\kappa_{j}}\right)=\frac{\Delta P \kappa_{j}-P_{j} \Delta \kappa}{\kappa_{j}^{2}+P_{j}^{2}} .
$$

Due to $\Delta \kappa=\kappa_{j}-\kappa_{j}=0$ and $\Delta P=P_{j+1}-P_{j}$, Eq.(7) reduces to a first order differential equation

$$
\frac{d P(x)}{d x}=\kappa^{2}(x)+P^{2}(x),
$$

where

$$
P(x)=-\frac{\psi^{\prime}(x)}{\psi(x)},
$$

which is a well-known Riccati equation. Substitution Eq.(9) into Eq.(8) results in the time-independent Schrödinger equation $\psi^{\prime \prime}(x)+\kappa^{2} \psi(x)=0$. From another angle, the deducing of Eq.(8) is proven to be true.
Likewise, Eq.(5) can be integrated as

$$
\delta(n)=\int_{x_{L}}^{x_{R}}\left(-\frac{d \kappa}{d x} \frac{P}{P^{2}+\kappa^{2}}\right) d x=\int_{x_{L}}^{x_{R}}\left(-\kappa^{\prime} \frac{P}{P^{\prime}}\right) d x .
$$

where $\kappa^{\prime}=d \kappa / d x$ and $P^{\prime}=d P / d x$. From Eq.(9), we obtain the wave function

$$
\psi(x)=N_{0} \exp \left[-\int^{x} P\left(x^{\prime}\right) d x^{\prime}\right],
$$

which is acquired to be square integrable. In order to determine $P(x)$, one must solve Eq.(8). The initial condition of Eq.(8) is determined from $P_{D}$, while the function $\psi(x)=N_{1} \exp \left[-\alpha\left(x-x_{D}\right)\right]$ is matched to the wave function decaying exponentially in the range $\left[x_{D}, \infty\right]$, with $\alpha=\sqrt{2 m\left[V\left(x_{D}\right)-E\right]}$ which is the equivalent attenuated vector, so $P_{D}=-\psi^{\prime}\left(x_{D}\right) / \psi\left(x_{D}\right)=\alpha$.

Now from Eq.(4) and Eq.(10), i.e., $\int_{x_{L}}^{x_{R}} \kappa d x+\delta(n)=$ $(n+1) \pi$, we have derived the exact quantization condition

$$
\oint_{C}\left(\kappa-\kappa^{\prime} \frac{P}{P^{\prime}}\right) d x=2(n+1) \pi, n=0,1,2 \ldots,
$$

where the integral is counterclockwise around a closed contour $C$ which encloses the two turning points. The quantization condition complies with the requiring the total phase during one period of oscillation to be an integral multiple of $2 \pi$ for a particle oscillating in the classically allowed region between the two turning points $\left[x_{L}, x_{R}\right]$. Comparing with scattering theory in which the sub-waves phase is shifted when the particle is scattered by a potential, we can gain the better understanding why there is a term $\delta(n)$ in Eq.(12), which is ignored by other approximations such as the WKB approximation and the Bohr-Sommerfeld quantization condition.

In contrast with the classical Hamilton-Jacobi theory, the quantum action-angle variable eigenvalues $J$ should be defined as $J=J(E)=(1 / 2 \pi) \oint_{C} K d x$ with $K=\kappa-\kappa^{\prime} P / P^{\prime}$. Since $-\kappa^{\prime} P / P^{\prime}$ has the same momentum dimension as $\kappa, K$ is referred to as the complete quantum momentum function(QMF). The point is emphasized that the $J(E)$ is defined at arbitrary energies, not only the eigenvalues. The wave function outside the classically allowed region, corresponding to whether the energy eigenvalues or other arbitrary energies, cannot be exponential growth, on the contrary, is bound to decay exponentially because the potential $V(x)$ is larger than the particle $E$ outside the classically allowed region, the particle cannot fully enter the classically forbidden region, implying the wave function should decay exponentially. As a result, there is no the points where log derivative $P$ diverges. Considering the exponentially decaying wave function outside the classically allowed region and with the help of the Riccati Eq.(8), $J(E)$ in Eq.(12) can be calculated for arbitrary $E$, clearly the behavior of $J(E)$ is monotonic in all $E$. The new definition 
of $J$ is a conceptual breakthrough not only because no one reached it from the founding of quantum mechanics to now but also Eq.(12) possesses the basis going back to the Bohr-Sommerfeld quantization condition in the old quantum theory. Thereby, a one-to-one correspondence between the invariant torus and quantum eigenvalues is unambiguously established.

It should be noted that our defined $J(E)$ is a real function which describes the system motion, and, in addition, enables one to complete Hamilton's original program of associating a wave with a particle motion. In essence, the scattering of a particle in the bound state alters the momentum of the particle, as a result, contributes significantly to the exactness of Eq.(12). At the same time, Eq.(12) indicates the energy eigenvalue is involved both with the particle behavior between the two turning points and with the particle behavior outside the two turning points, which is the different aspect between the classical and quantum mechanics, while the condition $J(E)=$ $n+1$ yields the exact energy eigenvalues, the number 1 comes from the contribution due to the phase shift at the turning points. In view of the Einstein-Brillouin-Keller (EBK) quantization condition [7] [8], since it only includes the particle behavior between the two turning points, it is impossible to generally give birth to the exact results.

It is well worth mentioning the Bohm's hidden variable theory [9]. A pair of equations is obtained, in which one of terms is called quantum potential, while we define a QMF $K$ in Eq.(12). Apparently whether the so-called quantum potential or the QMF is related to the wavefunction. According to Bohm's theory Newton's second law can be written as $m d^{2} \mathbf{r} / d t^{2}=-\left.\nabla(V+Q)\right|_{r=r(t)}[10$, where $V$ is classical potential and $Q$ is the quantum potential. But in our theory the classical $\kappa$ and quantum momentum $-\kappa^{\prime} P / P^{\prime}$, namely, the $\mathrm{QMF} K$, contribute to the quantum dynamics of the system described by $m d \mathbf{r} / d t=\kappa-\kappa^{\prime} P /\left.P^{\prime}\right|_{r=r(t)}$. This detailed investigation will appear elsewhere.

The quantization condition Eq.(12) together with Eq.(8)-Eq.(11) is exact both for the solvable potentials and for the unsolvable potentials, in order to illustrate them explicitly, let us first examine the detailed values of the scattering-led phase shift and detailed expressions of the phase integral for several familiar solvable potentials. Why these potentials are chosen is that their analytical wave functions are known so that one can calculate $\delta(n)$, consequently, one can confirm the exactness of Eq.(8)Eq.(12).

(1). One-dimensional infinite square well $V(x)=0$ at $x \subseteq[0, L]$ but $\infty$ at other $x$. Due to $d \kappa / d x=0$, one has $\delta(n)=0$ and the quantization condition reads

$$
\int_{0}^{L} \sqrt{2 E} d x=(n+1) \pi, n=0,1,2 \ldots
$$

which yields exact energy eigenvalues $E_{n}=(n+1)^{2} E_{0}$

\begin{tabular}{cccc}
\hline \hline$n$ & $E^{\text {exact }}$ & $E^{\text {present }}$ & $E^{\text {Maslov }}$ \\
\hline 0 & -0.97815416 & -0.97815416 & -0.97834291 \\
1 & -0.935566613 & -0.93556613 & -0.93566866 \\
2 & -0.87203511 & -0.87203511 & -0.87210568 \\
3 & -0.78795362 & -0.78795362 & -0.78800723 \\
4 & -0.68386490 & -0.68386490 & -0.68390852 \\
5 & -0.56051533 & -0.56051533 & -0.56055291 \\
6 & -0.41901295 & -0.41901295 & -0.41904703 \\
7 & -0.26131274 & -0.26131274 & -0.09251698 \\
8 & -0.09248716 & -0.09248716 & -0.09251698 \\
\hline \hline
\end{tabular}

TABLE I: Comparisons of exact, present, and the Maslov index energy eigenvalues for the radial Woods-Saxon potential $V(r)=-\frac{1}{1+\exp [2(r-30)]}+\frac{l(l+1)}{2 r^{2}}$ with $l=1$, setting $m=\hbar=1$.

with $E_{0}=\pi^{2} \hbar^{2} / 2 L^{2}$. However, the WKB approximation $\int_{0}^{L} \sqrt{2 E} d x=(n+1 / 2) \pi \hbar$ can not do so.

(2). One-dimensional harmonic oscillator $V(x)=$ $x^{2} / 2$, whose eigenfunction can be expressed by elementary functions, i.e., $\psi_{0}=\sqrt{\alpha} / \pi^{1 / 4} \exp \left[-\alpha^{2} x^{2} / 2\right]$, $\psi_{1}=\sqrt{2 \alpha} / \pi^{1 / 4} \alpha x \exp \left[-\alpha^{2} x^{2} / 2\right] \ldots$, substituting $\psi_{n}$ into Eq.(8) and Eq.(10), we find $\delta(n)=\pi / 2$, to a certain degree, which shows Eq.(10) is exact because Eq.(12) reduces to the familiar WKB quantization condition that remains exact for the one-dimensional harmonic oscillator. For the three-dimensional harmonic oscillator $V_{\text {eff }}(r)=r^{2} / 2+l(l+1) / 2 r^{2}$, substituting the known wave functions into Eq.(8) and Eq.(10), we have $\delta(n, l)=$ $[2 \sqrt{l(l+1)}-(2 l-1)] / 4$, and the quantization condition reads

$$
\int_{r_{L}}^{r_{R}} \kappa d r+\frac{2 \sqrt{l(l+1)}-(2 l-1)}{4} \pi=(n+1) \pi,
$$

which yields the exact energy eigenvalues $E=2 n+l+$ $3 / 2 . \kappa$ is defined by $\kappa=\sqrt{2 m\left[E-V_{\text {eff }}(r)\right]}$ in Eq.(14). For this case, the total Maslov index 3$] \mu$ happens to accord with the term $2 l-2 \sqrt{l(l+1)}+3$. (3). Onedimensional Coulomb potential $V(x)=-1 /|x|$. Though there is a singularity at $x=0$ [11], in the similar way, we find $\delta(n)=\pi$, and the quantization condition reads

$$
\int_{0}^{x_{R}} \kappa d x=n \pi, n=1,2,3 \ldots
$$

where $x_{R}=-1 / E$. Eq.(14) gives the exact energy eigenvalues $E_{n}=-1 /\left(2 n^{2}\right)$. For the three-dimensional Coulomb potential $V_{\text {eff }}(r)=-1 / r+l(l+1) / 2 r^{2}$, we find $\delta(n, l)=[\sqrt{l(l+1)}-l] \pi$, and the quantization condition reads

$$
\int_{r_{L}}^{r_{R}} \kappa d r+[\sqrt{l(l+1)}-l] \pi=(n+1) \pi, n=0,1,2 \ldots
$$




\begin{tabular}{cccc}
\hline \hline$n$ & $E^{\text {exact }}$ & $E^{\text {present }}$ & $E^{\text {SWKB }}$ \\
\hline 0 & 14.312470 & 14.312470 & 14.312670 \\
1 & 14.468822 & 14.468822 & 27.174940 \\
2 & 42.227424 & 42.227424 & 39.499957 \\
3 & 43.899629 & 43.899629 & 47.940588 \\
4 & 68.213256 & 68.213256 & 62.315504 \\
5 & 74.975448 & 74.975448 & 76.652144 \\
6 & 94.567829 & 94.567829 & 92.325304 \\
7 & 108.464359 & 108.464359 & 108.985251 \\
8 & 124.328691 & 124.328691 & 126.433812 \\
\hline \hline
\end{tabular}

TABLE II: Comparisons of exact, present, and SWKB energy eigenvalues for the double oscillator $V(x)=10(|x|-3)^{2}$, setting $m=\hbar=1$.

which determines the exact energy eigenvalues $E=$ $-1 /\left(2 N^{2}\right)$ with $N=l+n+1$.

Eqs.(13)-(16) are novel and immediate expressions under no other auxiliary modifications, whose exactness can make one convinced that the scattering-led phase shift $\delta(n, l)$ does exist objectively, and it plays a crucial role in determining the energy eigenvalues. Because of it, when the WKB approximation is applied to the three-dimensional potentials, Langer-correction is needed. Interestingly, if the number $l(l+1)$ is instead of $(l+1 / 2)^{2}$ in Eq.(14) and Eq.(16), they are naturally changed to Langer-correction formalism $\int_{x_{L}}^{x_{R}} \sqrt{2 m\left[E-V_{e f f}(r)\right]} d r=(n+1 / 2) \pi$ with $V_{\text {eff }}(r)=$ $V(r)+(l+1 / 2)^{2} / 2 r^{2}$. Moreover, when the Coulomblike potentials is tackled, the problem of singularity is naturally avoided. With regard to the unsolvable potentials, generally $\delta(n, l)$ is not an analytical expression of $n$ and $l$. Next the energy eigenvalues for the unsolvable radial Woods-Saxon potential 12 and the unsolvable double oscillator potential [13] with Eq.(12) are tabulated in Table I and II, respectively, and the complete agreement with exact results can be seen, implying Eq.(12) is exact. $E^{\text {exact }}$ is obtained through numerical technique. Dealing with the double oscillator potential, as the energy of the particle is relatively smaller, four turning points can occur, assuming $x_{1}<x_{2}<x_{3}<x_{4}$. Because the particle will be motioning in the range $\left[x_{1}, x_{4}\right]$, the quantization condition $\int_{x_{1}}^{x_{4}}\left(\kappa-\kappa^{\prime} P / P^{\prime}\right) d x=(n+1) \pi$ gives the energy eigenvalues. For other complex potentials with more then four turning points, in the similar way, the contour is taken from the smallest turning point to the biggest turning point. In these cases, the tunneling effect takes place.

In summery, based on the transfer matrix rather than the wave function expansion in powers of $\hbar$, we have derived the exact quantization condition for arbitrary nonrelativistic quantum systems and have shown it unifies and transcends the historical approaches. Naturally, we have obtained the corresponding real quantum actionangle variable, contrasted with the classical HamiltonJacobi theory, which discloses the quantum-classical correspondence.

This work is supported by National Natural Science Foundation of P.R.China under grant No.60237010 Municipal Scientific and Technological Development Project of Shanghai under grant No.012261021, 01161084.

* Electronic address: ouyongcheng@163.com

[1] R. E. Langer, Phys. Rev. 51, 669(1937).

[2] S. C. Miller and R. H. Good, Phys. Rev. 91,174(1953).

[3] H. Friedrich and J. Trost, Phys. Rev. Lett. 76, 4869(1996).

[4] A. Comtet, A. D. Bandrauk, and D. K. Campbell, Phys. Lett. B 150, 159(1985).

[5] D. T. Barclay, A. Khare and U. Sukhatme, Phys. Lett. A 183, 263(1993).

[6] Z. Q. Cao, Y. Jiang, Q. S. Shen, X. M. Dou and Y. L. Chen, J. Opt. Soc. Am. A 16, 2209(1999).

[7] L. S. Schulman, Technigues and Applications of Path Integration (Wiley, New York, 1987).

[8] P. Gaspard, D. Alonso, and I. Burghardt, Adv. Chem. Phys. 90, 105(1995).

[9] D. Bohm, Phys. Rev. 85, 166(1952); 85, 180(1952).

[10] O. F. de Alcantara Bonfim, Phys. Rev. E 58, 2693(1998).

[11] J. Hainz and H. Grabert, Phys. Rev. A 60, 1698(1999).

[12] E. Koch, Phys. Rev. Lett. 76, 2678(1996).

[13] B. Chakrabarti and T. K. Das, Phys. Rev. A 60, 104(1999). 